\title{
MEASUREMENTS AND CALCULATIONS OF THE CIRCULAR POLARIZATION AND OF THE ABSOLUTE INTENSITY OF SYNCHROTRON RADIATION IN THE WAVELENGTH RANGE FROM 40 TO $100 \mathrm{~nm}$
}

\author{
U. HEINZMANN, B. OSTERHELD and F. SCHÄFERS \\ Fritz-Haber-Institut of MPG, D-1000 Berlin 33, Faradayweg 4-6, Synchrotron of the University Bonn, Fed. Rep. Germany
}

\begin{abstract}
The circular polarization and the absolute intensity of the elliptically polarized synchrotron radiation were measured in the VUV range as functions of the wavelength and of the vertical emission angle with respect to the orbit plane of the $2.5 \mathrm{GeV}$ synchrotron in Bonn. In the wavelength range from 100 to $40 \mathrm{~nm}$ the absolute intensity and the circular polarization of the radiation with a bandwith of $0.05 \mathrm{~nm}$ has been experimentally determined to be between $10^{9}$ and $10^{10}$ photons $s^{-1}$ and $(83 \pm 3) \%$, respectively. The experimental data are compared with calculated results obtained on the basis of Schwinger's theory taking into account the properties of the synchrotron as well as of the components in the $10 \mathrm{~m}$ normal incidence monochromator.
\end{abstract}

According to Schwinger's theory synchrotron radiation is linearly polarized in the plane of the electron beam; the radiation emitted into directions above and below it is elliptically polarized with a high fraction of circular polarization. The circular polarization of the VUV radiation of the Bonn-synchrotron has been used for measurements of the Fano effect at atoms and molecules [1] (spin transfer from photons to photoelectrons in the photoionization with circularly polarized light); for this new field of research it was necessary to know the degree of circular polarization.

A schematic diagram of the apparatus used is shown in fig. 1 . The synchrotron radiation passes through a $10 \mathrm{~m}$ normal incidence monochromator with a plane holographic grating (4960 lines $\mathrm{mm}^{-1}$ ) and a concave mirror, which produces an image of the electron beam in the exit slit. The angular range accepted is $20 \mathrm{mrad}$ and $\pm 3.5 \mathrm{mrad}$ in the horizontal and vertical direction, respectively. The radiation coming from the electron beam is cut off in vertical direction by an aperture which is movable up and down for selecting radiation of lefthanded (upper half) or right-handed (lower half) circular polarization, respectively.

The circular polarization of the monochromated radiation (bandwidth $0.05 \mathrm{~nm}$ ) has been analyzed by a rotatable arrangement of four gold coated mirrors (reflection at $60^{\circ}$ ). By means of the four-fold reflections an analyzing power of more than $99 \%$ has been achieved. Because this analyzer cannot distinguish between circularly polarized and unpolarized radiation, in an additional measurement the overall polarization had to be measured: a quarter wave plate $\left(\mathrm{MgF}_{2}\right.$ at $150 \mathrm{~nm}$ [2]) not shown in fig. 1 has been used to transform the elliptical polarization into a linear one, which is then analyzed by the four gold mirrors. By this method the unpolarized background radiation has been determined to be smaller than $1 \%$, which means that the overall degree of polarization is

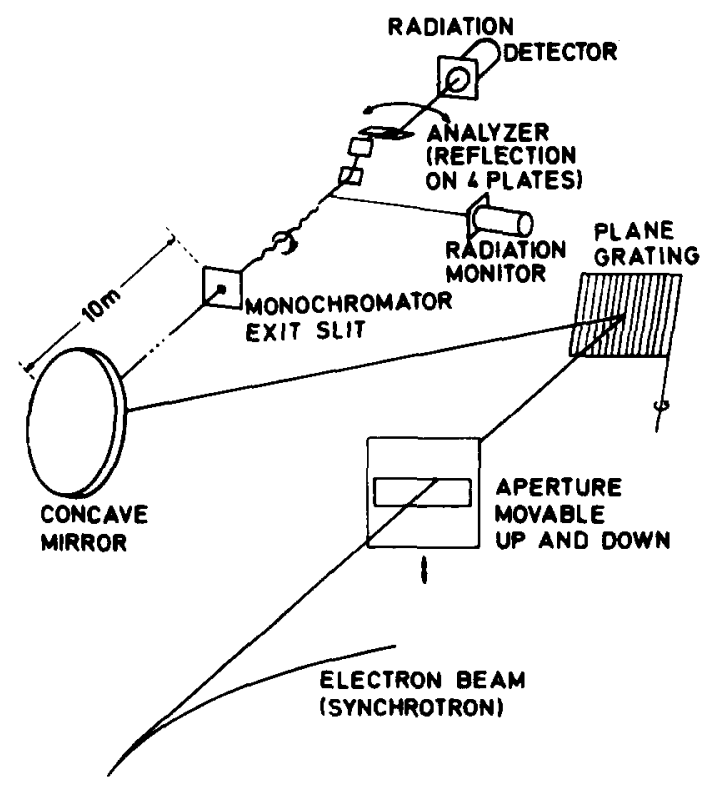

Fig. 1. Schematic diagram of the apparatus. 
one for synchrotron radiation. The radiation transmitted through the mirror-arrangement has been detected by an open multiplier; a second multiplier has been used as monitor in order to record instabilities of the light intensity.

For measurements of the absolute intensity of the circularly polarized VUV radiation the analyzer shown in fig. 1 was replaced by a double ionization chamber of the Samson type [3] not shown in fig. 1. The chamber was placed $0.8 \mathrm{~m}$ behind the exit slit of the monochromator; the light spot was thus about $8 \times 20 \mathrm{~mm}^{2}$, scanned by moving the whole chamber $\left(2 \times 2 \mathrm{~mm}^{2}\right.$ entrance-hole $)$ horizontally and vertically. All measurements have been performed at an energy of $0.8 \mathrm{GeV}$ at the 2.5 $\mathrm{GeV}$ synchrotron and with a duty cycle of 0.6 (dedicated mode, accelerated and decelerated eletron beam); all intensities shown are normalized to a typical electron current of $60 \mathrm{~mA}$.

Fig. 2 shows the dependence of the absolute intensity on the vertical position of the ionization chamber for the wavelength of $100 \mathrm{~nm}$. Error bars are shown at four points only.

The experimental results (points) show that the radiation is emitted into a small vertical range

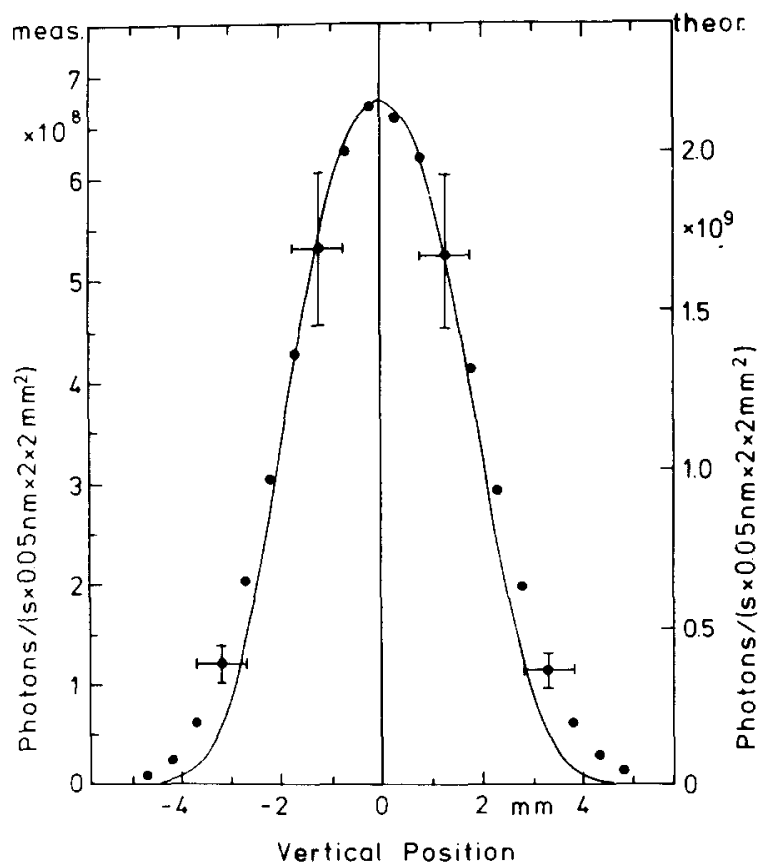

Fig. 2. Vertical profile of the absolute intensity of $100 \mathrm{~nm}$ synchrotron radiation: experimental results (error bars and left scale) and theoretical curve (rigth scale). (about $3 \mathrm{mrad} f w h m$ ). The profile is symmetric with respect to the plane of the synchrotron (position 0 ) but does not show the characteristic minimum in the plane. There are two ordinates in fig. 2, the left one corresponding to the values measured (points) and the right one corresponding to the solid curve calculated. They coincide within a factor of three.

The theoretical curve was determined ab initio following the theory of Schwinger [4], taking into account the real differences between a storage ring and a synchrotron, the height of the electron beam of $1 \mathrm{~mm}$ (details of the procedure are published elsewhere [5]), the reflectances of the platinum coated grating [6] and mirror [7] and the width of the entrance slit of the chamber. The agreement of the values measured with the theoretical curve is quite good, taking into account the uncertainties in the reflectances of the optical components and the fact that the measurements have been performed two years later after the components were built in the monochromator. During the measurement just described no aperture shown in fig. 1 was in the light beam. By integrating the profile together with the corresponding horizontal profile the total intensity at the exit slit can be obtained.

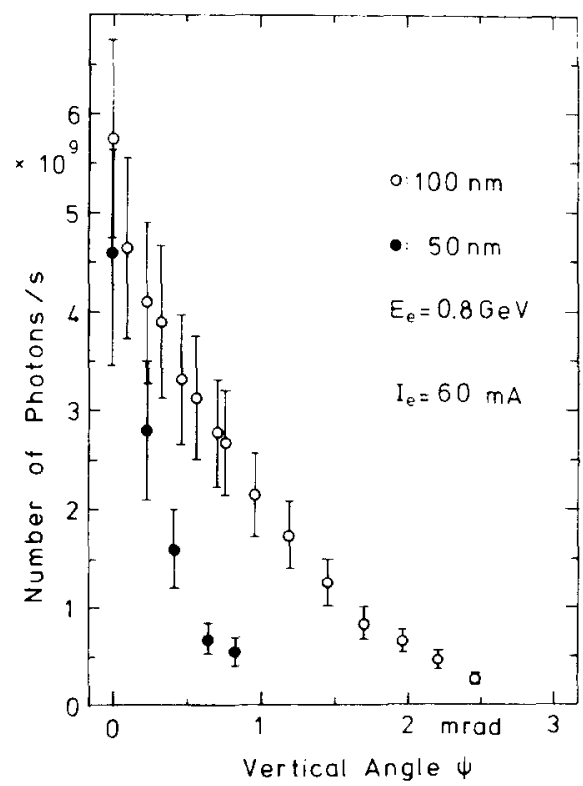

Fig. 3. Dependence of the total absolute intensity on vertical angle $\psi$ for radiation emitted into a vertical angular range from $\psi$ to $3.5 \mathrm{mrad}$ : experimental values (error bars) for two wave lengths 


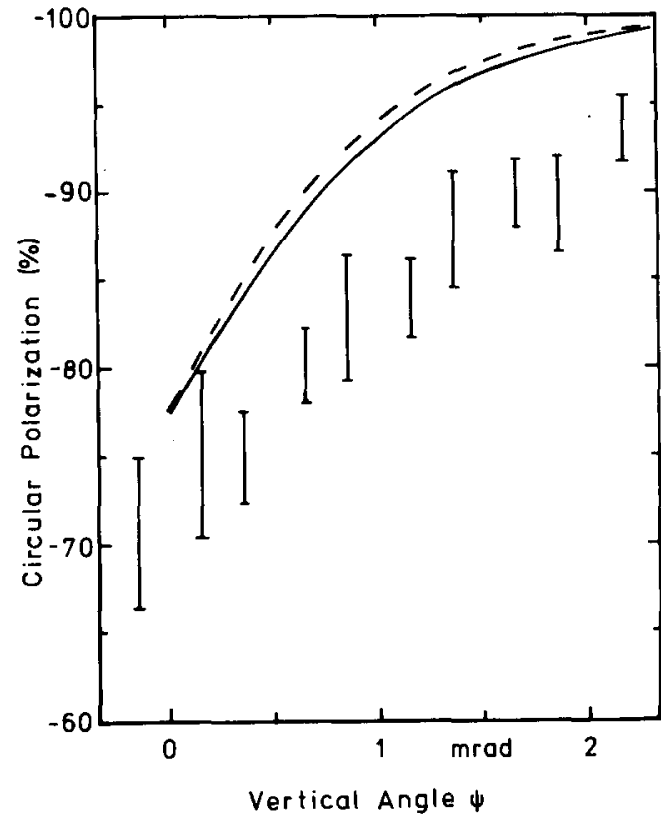

Fig. 4. Dependence of the circular polarization of sychrotron radiation on the vertical angular range accepted from $\psi$ to 3.5 mrad: experimental results (error bars) and theoretical calculations (broken curve: height of electron beam zero; solid curve: height of electron beam $1 \mathrm{~mm}$ ).

For different positions of the aperture, which cuts off a certain amount of radiation in the vertical direction, the total intensity was determined and is plotted in fig. 3 versus the position of the aperture for two wavelengths ( 100 and 50 $\mathrm{nm})$. Each error bar gives the absolute intensity of radiation emitted into a vertical angular range from $\psi$ to $3.5 \mathrm{mrad}$. It can be easily seen that the angular range over which the radiation is emitted becomes smaller with shorter wavelengths.

The corresponding circular polarizations measured are shown in fig. 4 for a wavelength of 100 $\mathrm{nm}$ and an electron energy of $0.8 \mathrm{GeV}$. The polarization is drawn as a function of the vertical angle $\psi$ with respect to the synchrotron plane, where the vertical angular range accepted is from $\psi$ to 3.5 mrad. As is shown in fig. 4 , the circular polarization of all the radiation emitted in all upper directions with respect to the plane (position 0 in fig. 4) has an average value of $-70 \%$. The polarization increases with increasing vertical angle up to nearly $-100 \%$ whereas the intensity decreases rapidly to zero (fig. 3).

The dotted curve in fig. 4 represents the degree

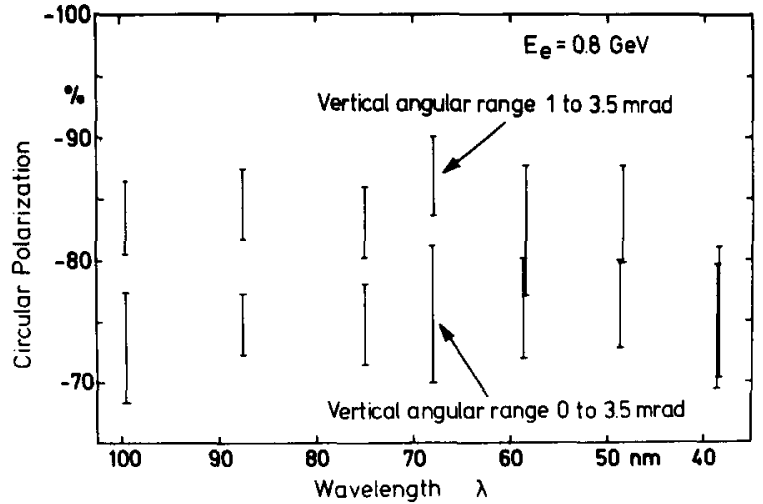

Fig. 5. Wavelength dependence of the circular polarization for radiation emitted into two vertical angular ranges.

of circular polarization of radiation emitted by the Bonn-synchrotron, not taking into account the height of the electron beam, calculated ab initio following Schwinger's formulae. The full curve has been theoretically obtained assuming a homogeneous "smearing out" of the electron beam at a height of $1 \mathrm{~mm}$. The difference between the theoretical curves and experimental results in fig. 4 is about $10 \%$, which arise mainly as a result of the betatron and synchrotron oscillations not taken into account in the theory. It is worth noting that the optical components should only have a negligible influence on the light polarization, because normal incidence reflection and Littrow mounting have been used for the mirror and grating, respectively.

The results of the wavelength dependence of the

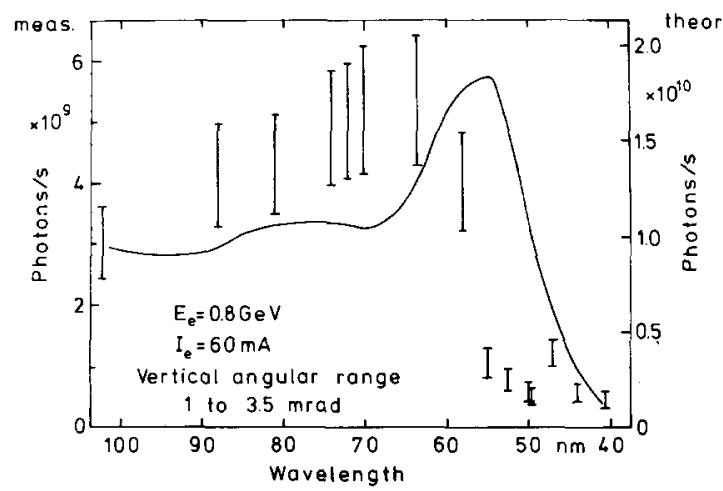

Fig. 6. Wavelength dependence of the absolute intensity of circularly polarized synchrotron radiation emitted into the vertical angular range from 1 to $3.5 \mathrm{mrad}$ behind the exit slit of the $10 \mathrm{~m}$ monochromator: experimental results (error bars and left scale) and calculated curve (right scale). 
circular polarization measured are shown in fig. 5 in the wavelength range from 40 to $100 \mathrm{~nm}$ in the vertical angular range from 1 to $3.5 \mathrm{mrad}$ (upper errors bars) and from 0 to $3.5 \mathrm{mrad}$ (lower error bars). As shown the circular polarization is almost wavelength-independent, $(-83 \pm 3) \%$ and $(-75 \pm$ $4) \%$ in both angular ranges, respectively. Combining these results with the data on absolute intensity (fig. 3), only $20 \%$ of the total intensity at a wavelength of $100 \mathrm{~nm}(5 \%$ at $50 \mathrm{~nm}$ ) emitted into the whole vertical angular range can be used in an experiment if a circular polarization of more than $80 \%$ is needed.

In fig. 6 the wavelength dependence of the intensity of VUV-radiation emitted into the vertical angular range from 1 to $3.5 \mathrm{mrad}$ with respect to the synchrotron plane is shown. There are two ordinates again, the left one corresponding to the error bars of the experimental values and the right one corresponding to the calculated curve. The qualitative agreement between theory and experiment with respect to the intensity of the radiation behind the exit slit of the $10 \mathrm{~m}$ monochromator is quite good. There are two discrepancies, firstly the factor of three in the absolute scale mentioned above and secondly a small shift of the peak at about $60 \mathrm{~nm}$, which has its origin in the wavelength dependence of the reflectances of the grating and mirror.

We have shown that the polarization and the absolute intensity of synchrotron radiation can be calculated ab initio with good agreement with the corresponding experimental results, even if the radiation is emitted by a synchrotron and if all the properties of a monochromator are taken into account. Intensity and circular polarization are high enough for spin-transfer experiments in the photoionization of atoms and molecules, i.e. the synchrotron radiation emitted off axis with respect to the plane has been experimentally verified to be a powerful source of circularly polarized VUVradiation.

The authors wish to express their graditude to the Physikalisches Institut of the Universität at Münster and of the Universität at Bonn for their support of the project performed at the Bonn synchrotron. Support by the Bundesminister für Forschung and Technologie is gratefully acknowledged.

\section{References}

[1] For a review, see U. Heinzmann, Appl. Opt. 19 (1980) 4087.

[2] U. Heinzmann, J. Phys. E: 10 (1977) 1001.

[3] J.A.R. Samson, J.O.S.A. 54 (1964) 6.

[4] J. Schwinger, Phys. Rev. 75 (1949) 1912.

[5] B. Osterheld, Diploma Thesis, University of Münster (1981).

[6] M. Nevière, private communication (1977).

[7] A. Seignac and S. Robin, Sol. St. Commun. 11 (1972) 217. 